\title{
A comparative study of postgraduate medical education in North East Thames Region
}

\author{
A. Barker ${ }^{1}$, A.D. Scotland ${ }^{2}$, S. Challah ${ }^{1}$, B. Gainey ${ }^{2}$ and I. Bayley ${ }^{3}$ \\ ${ }^{1}$ Touche Ross Management Consultants, ${ }^{2}$ North East Thames Regional Health Authority and ${ }^{3}$ Royal \\ National Orthopaedic Hospital, Stanmore, Middlesex, UK
}

\begin{abstract}
Summary: As a prelude to more detailed formal contracting, North East Thames Region undertook a review to examine whether the content of postgraduate medical education (PGME) varies according to the type of hospital in which junior doctors are trained. The study covered a sample of 83 trainees at different grades in four types of hospital (postgraduate, university, district general hospital involved in off-site undergraduate medical education, and district general hospital with no formal involvement in undergraduate medical education) and was designed as a qualitative comparative study. The results of the study point to a perceived lack of structure in PGME and indicate that hospital type alone does not determine a trainees' PGME experience. Moreover, different training grades have different educational needs, which will need to be addressed under more formal contracting arrangements. The Region plans to take this work forward by convening one or more consensus conferences to examine how a more structured approach to PGME could be implemented.
\end{abstract}

\section{Introduction}

Medical education in the United Kingdom is undergoing a period of review and change. The changes include:

- The publication of a review by the Chief Medical Officer, which proposes significant changes to the way doctors are trained for consultant posts. ${ }^{1}$

- The review of research in Special Health Authorities undertaken by the Review Advisory Committee published in June 1993. ${ }^{2}$

- From April 1993, the introduction of changes to funding of approved training posts. Regional Health Authorities now meet $50 \%$ of salary costs and all non-salary costs.

- The allocation of budgets to clinical tutors, covering, for example, study leave and facility costs.

In the context of these changes, and in the light of the requirements for contracting, North East Thames Regional Health Authority undertook a review to examine whether the content of postgraduate medical education (PGME) varies according to the type of hospital in which junior doctors are trained. The region commissioned

Correspondence: A. Scotland, F.R.C.S.(Ed.), F.F.P.H.M., North Thames Regional Health Authority, 40 Eastbourne Terrace, London W2 3QR, UK.

Accepted: 3 May 1994
Touche Ross Management Consultants to carry out the research work and the authors of this report comprised the project team. The study covered senior house officers (SHOs), registrars and senior registrars in four types of hospital: postgraduate hospital; university hospital; district general hospital (DGH) involved in off-site undergraduate medical education (DGH I); and DGH with no formal involvement in undergraduate medical education (DGH II).

The expectation was that trainees' PGME experience would reflect the differences in the clinical and academic orientation of each type of hospital.

Although not directly concerned with the financing and organizational changes under discussion for medical education, the study's findings were intended to assist the region to move towards more structured arrangements for resourcing and monitoring PGME, as a prelude to better formal contracting.

The exercise was not intended as an audit of the educational activities undertaken at the same hospitals. To reinforce this, the findings have been made anonymous.

\section{Methods}

The study was conducted using a semi-structured questionnaire administered in face to face inter- 
views in the four hospitals. The study was designed as a qualitative comparative study. The results were not intended to be statistically significant.

A pilot study for the questionnaire was conducted in one hospital in late January 1993. (Copies of the questionnaire are available on request from the authors.) The main study interviews were held over a 5-week period from mid-February to midMarch 1993.

\section{Sample}

Consultants in each hospital were asked to nominate trainees for interview but no attempt was made formally to stratify the sample by grade or by specialty. Instead of formal stratification, we specified for each hospital: a fixed number of interviews, relative to the total number of trainees in each grade; and that the sample should include trainees from a representative range of specialties.

A total of 83 trainees were interviewed during the course of the study, representing just over a quarter of all doctors across the three grades in the four study hospitals.

The doctors in the sample represent a broad range of acute specialties. Overall, one third of the trainees interviewed were from surgical specialties and a further $28 \%$ were from medical specialties. The remainder were from obstetrics and gynaecology $(7 \%)$, paediatrics $(7 \%)$, accident and emergency $(5 \%)$, anaesthetics $(12 \%)$, pathology $(7 \%)$ and psychiatry $(1 \%)$. We have drawn limited conclusions on a specialty basis, however, because the numbers for most specialties are small and the distribution of trainees by specialty between hospitals and grades is not even.

\section{Results}

The questionnaire covered a number of issues. The results presented below include the main areas reviewed.

\section{Working arrangements}

Although we recognize that many trainees work longer hours than those formally specified in their contracts, contracted hours were requested as a basis for comparison between the four hospitals (Table I).

Trainees at the postgraduate hospital had fewer contracted hours and lower on-call commitments. This was particularly the case for senior registrars and registrars, but less so for SHOs, who had a greater contracted hours commitment.

There was much less difference in average contracted hours and on-call commitments between the university hospital and the two DGHs. Overall, trainees in DGH I worked fewer contracted hours on average than their counterparts at the university hospital and DGH II, and were significantly less likely to work a one in three rota. SHOs in DGH I had the lowest average contracted hours of all the four hospitals.

The vast majority of trainees were on rotation, with little difference in this respect between the four hospitals.

\section{Pastoral care}

Questions were based on the region's objectives in this area. Trainees were asked to describe the arrangements for induction, the availability of a training prospectus (setting out the training opportunities offered), the availability of a formal mentor or educational supervisor charged with overseeing trainees' educational experience and the arrangements for formal progress review.

We found few consistent differences between the four hospital types. Most trainees $(80 \%)$ did not receive induction and $77 \%$ did not receive a training prospectus. In addition, only $35(42 \%)$ were aware of a formal mentor. Formal review was a key area of dissatisfaction among trainees in all four hospitals.

The study indicates that Regional objectives for pastoral care are not currently being met. We found little evidence of individual goal setting or performance review for PGME, and accordingly, the systems (such as formal appraisal) to support this were not widely available. There was, however, some evidence that DGHs have adopted a more structured approach than teaching hospitals in this regard, particularly for SHOs. SHOs in the two DGHs appeared to receive more structured induc-

Table I Average contracted additional duty hours (ADHs) by grade and hospital

\begin{tabular}{lcccccc}
\hline Hospital & $\begin{array}{c}\text { Senior registrar } \\
\text { (hours) }\end{array}$ & (Ranking) & $\begin{array}{c}\text { Registrar } \\
\text { (hours) }\end{array}$ & (Ranking) & $\begin{array}{c}\text { SHO } \\
\text { (hours) }\end{array}$ & (Ranking) \\
\hline Postgraduate & 21.6 & $(4)$ & 26.0 & $(4)$ & 33.0 & $(3)$ \\
University & 35.8 & $(1)$ & 41.8 & $(2)$ & 37.8 & $(2)$ \\
DGH I & 33.1 & $(2)$ & 32.2 & $(3)$ & 30.9 & $(4)$ \\
DGH II & 32.0 & $(3)$ & 43.0 & $(1)$ & 44.5 & $(1)$ \\
\hline
\end{tabular}

Note: a ranking of 4 indicates the lowest average ADHs and a ranking of 1 the highest. 
tion (related to specialty), mentorship and progress review.

\section{Facilities}

Trainees were asked whether they had access to a postgraduate centre and library facilities, and whether the library facilities were adequate for their needs.

The university hospital was the only hospital without a dedicated postgraduate facility. This was not, however, perceived to be a problem as PGME activities were either held in departmental or Medical School facilities. In the other three hospitals, a dedicated postgraduate education centre was available on the main site.

Outside the postgraduate hospital, where all trainees regarded the library facilities as adequate or more than adequate, the perceived adequacy of library facilities did not appear to be related to hospital type. In the university hospital, $25 \%$ of respondents rated library facilities as inadequate compared to $43 \%$ in DGH I and $11 \%$ in DGH II.

\section{Time available}

Trainees were asked about the arrangements for study leave and whether arrangements for formal protected education time had been introduced. In the main, trainee's study leave allowances coincided with national guidelines. However, administrative arrangements and the extent to which trainees could take study leave in practice varied.

The majority of trainees did not consider that they had access to protected education time. Moreover, the definition of protected education time was not universally shared. We draw this conclusion because the hospital which, in our view, came closest to having introduced protected education time was DGH I, but this was not reflected in the views of DGH I trainees. In fact, just under half of the SHOs and $40 \%$ of the registrars there did not consider they had access to protected time.

In contrast, at the postgraduate hospital, where education time is not formally protected, but senior grade trainees are encouraged to attend a full programme of events, all registrars and senior registrars reported that they had protected time. However, only one SHO out of the four interviewed at the postgraduate hospital reported regular attendance at the weekly education programme. At SHO level, therefore, DGH I appeared to offer the most protected time for trainees.

Some individual specialties in DGH II and the university hospital had introduced elements of protected education time, but there were no hospital-wide initiatives.

\section{Research}

Trainees were asked whether they were currently engaged in any research activities. There was a clear difference between the university/postgraduate hospitals and the DGHs, with trainees at the former institutions being more likely to be involved in research. This difference was particularly marked at registrar and SHO levels. For example, whereas all registrars and $50 \%$ of SHOs at the postgraduate hospital reported involvement in research, only one of the registrars $(17 \%)$ and none of the SHOs in DGH II were engaged in such activities. Similarly, although the proportion of trainees in DGH I ( $40 \%$ of registrars and $14 \%$ of SHOs) was higher than for DGH II, it was below the numbers reporting research involvement at the university at those two grades $(90 \%$ and $30 \%$, respectively).

\section{Clinical training}

Trainees were asked about training and education received in three clinical settings: ward rounds, outpatient clinics and operating theatres.

\section{Ward rounds}

Formally defined teaching rounds for PGME are uncommon. Very few trainees reported that they undertook ward rounds solely for teaching purposes and, where these did arise, it was often because the trainee was able to join an undergraduate teaching round.

Most consultant PGME teaching appears to be provided in general ward rounds (business rounds). The teaching element is rarely formally defined. For example, some trainees recorded that every ward round with a consultant potentially offered a teaching opportunity, whereas others only included those with a specific emphasis on teaching (for example, more time was allowed than in a general business round). Equally, other trainees considered that no consultant ward rounds offered any teaching opportunities.

There was a significant difference in the reported experience of trainees in the two DGHs; whereas $61 \%$ of ward rounds in DGH I were categorized as providing teaching, $71 \%$ in DGH II were categorized as business only. The variability in response appears to reflect both actual variation in experience, which is heavily dependent on the propensity of individual consultants to teach, and trainee's subjective categorization in the absence of a formal definition of PGME ward teaching. 


\section{Outpatient clinics}

Differences were apparent between the hospitals. The postgraduate hospital offered a higher proportion of clinics in which training was provided, i.e. where the trainee either only observed or saw patients in the same room as a consultant. Nearly a quarter of clinics were organized in this way. However, these opportunities are generally only open to registrars and senior registrars.

The outpatient teaching experience of trainees in the other three hospitals was not markedly different. Overall, trainees at the university hospital were less likely to receive direct supervision than trainees in the two DGHs. However, the composition of the university hospital sample may partly account for this, as it included a larger number of senior registrars, who might be expected to run their own clinics.

The results suggest that there is relatively little formal teaching in outpatients, with the possible exception of senior registrars and registrars at the postgraduate hospital. For the majority of trainees, educational opportunities within outpatients were ad hoc. Consistent with this, the most common criticism levelled by trainees was that they did not have the opportunity to examine, treat and learn about complex cases.

\section{Theatres}

There were clear differences between the postgraduate hospital and the other hospitals in our sample in respect of teaching in theatres. Compared to the other hospitals, the postgraduate hospital offered a relatively large proportion of observation only sessions $(19 \%)$ and a relatively low proportion of unassisted operating, notwithstanding the predominance of senior registrars in the interview sample. This was reported to be due to the specialist nature of the postgraduate hospital case mix.

There were other differences between the hospitals. In DGH II, there was a lower proportion of unassisted sessions than in DGH I or the university hospital. A contributory factor might be the number of anaesthetic trainees interviewed in DGH II. Anaesthetic trainees are generally subject to more formal supervision than their surgical counterparts. In DGH II they comprised $20 \%$ of the sample compared to around $10 \%$ in DGH I and the university hospital.

Although there appears to be a similar level of unassisted operating in DGH I and at the university hospital, there were differences in the grades of trainees to whom this applied. At the university hospital, only senior registrars reported that they worked completely unassisted. In contrast, in
DGH I, some registrars and SHOs worked without direct supervision.

The findings for supervision/instruction in theatres were consistent with our findings for the other two areas of clinical training. Overall, there is little formal scheduled instruction in theatres. On the whole, trainees learn by assisting consultants but this relies heavily on the willingness of individual surgeons to teach. Senior grades at the postgraduate hospital appear to have more opportunity than others to be taught on specialist cases. However, the prospects for SHOs there are less satisfactory than for their senior colleagues.

As for outpatients, trainees were most critical about the lack of opportunity to gain practical experience of operating. There is evidence that trainees in DGHs are more likely to gain hands-on experience than trainees in teaching hospitals, particularly at registrar and SHO grades. In general, we found that there were more welldefined supervision arrangements for anaesthetic trainees.

\section{Formal education}

Trainees were shown a list of possible formal educational activities and were asked to indicate which, if any, were available to them, how often the activities were held, their duration and location and by whom they were provided. The activities included grand rounds, other lectures, departmental meetings and journal clubs.

We found that the university and postgraduate hospitals provided a greater range of potential activities than the two DGHs. In particular, the teaching hospitals were more likely to hold grand rounds and to convene lecture programmes offering outside speakers.

Trainees in the postgraduate hospital also spent more hours per week engaged in formal education activities than trainees in the other three hospitals. In contrast, there was much less difference between the university hospital and the DGHs. Despite the greater availability of activities at the university hospital, trainees there were often unable to attend due to other commitments.

When the time spent on formal education was taken as a percentage of average contracted hours, the differences between the postgraduate hospital and the others became more marked (Table II).

\section{Satisfaction}

Trainees were asked to rate the training and education they received in their current posts. For senior registrars and registrars, trainees at the postgraduate hospital were more satisfied than their counterparts at the other hospitals. Senior registrars in the two DGHs appeared to be partic- 
Table II Average formal education times as a percentage of average contracted hours

\begin{tabular}{lccc}
\hline Hospital & $\begin{array}{c}\text { Senior registrar } \\
(\%)\end{array}$ & $\begin{array}{c}\text { Registrar } \\
(\%)\end{array}$ & $\begin{array}{c}\text { SHO } \\
(\%)\end{array}$ \\
\hline Postgraduate & 10.4 & 8.3 & 5.7 \\
University & 4.4 & 3.9 & 3.2 \\
DGH I & 3.1 & 4.3 & 3.8 \\
DGH II & 5.2 & 2.7 & 2.9 \\
\hline
\end{tabular}

ularly dissatisfied, although it is difficult to draw too many conclusions because the sample size was so small. The level of satisfaction reported by all SHOs showed less difference between the four hospitals. SHOs in DGH I were the most satisfied. This corresponds with our findings in other areas, which suggested that SHOs in DGH I worked fewer hours on average, had reasonable access to study leave and enjoyed a more structured approach to education.

\section{Conclusions}

The key findings from the study are:

1. Hospital type alone does not of itself determine a trainee's PGME experience. Although there were differences between the hopitals, these were not consistent across grades or specialties. The exception to this was the experience of registrars and senior registrars at the postgraduate hospital.

2. Different training grades have different educational needs, which may cut across hospital settings. In order to introduce more formal contracting arrangements for PGME, it may be necessary to address the needs of each grade separately.

3. Overall, there is a perceived lack of structure in PGME. Trainees commonly regarded the training and education they received as ad hoc and dependent on the individual consultant's willingness and ability to teach. This was the same both within and between hospitals, grade and specialties.

The factors that differentiated the PGME experience of registrars and senior registrars at the postgraduate hospital included:

1. Lower contracted hours and clinical commitments, allowing more time to focus on education and research.

2. The range, quality and quantity of formal education activities available.

3. The opportunity to pursue research.

4. The opportunity for instruction on specialist cases, facilitated by formal clinical training in outpatients and theatres in some instances.
5. An organizational emphasis on education and research, demonstrated by the priority placed on formal, timetabled educational activities.

However, although the postgraduate hospital appeared to offer registrars and senior registrars a qualitatively different experience, such differences did not extend to SHOs there. In addition, there was far less to differentiate the experience of trainees in the university hospital from that of their DGH counterparts. Indeed, at SHO level, there was some evidence that DGHs offered a more structured approach than either the postgraduate or the university hospitals.

The differences in educational needs by grade were particularly marked for SHOs. In general, SHOs require a more structured approach, involving clear benchmarking for skills and experience attainment, and are more focused on examinations than their senior colleagues, for whom such structure is less important.

Lack of structure in the provision of PGME was particularly noticeable in the lack of formally defined educational or training inputs in trainees' clinical activities on ward rounds, in outpatients and in theatres. For the majority of trainees, clinical training in these settings is gained in an ad hoc way. However, models of good practice do exist and it was noticeable that the postgraduate hospital appeared to afford greater opportunity for senior grades to obtain instruction on specialist cases.

Trainees themselves reported that there were differences between university/postgraduate hospitals and DGHs. Whereas university and postgraduate hospitals were felt to offer opportunities to manage complex or unusual cases, DGHs were perceived as places trainees could learn about general cases, and become skilled in treating them. Trainees recognized the value of all three main types of hospitals in their overall training.

However, notwithstanding these differences reported by trainees, we found trainees in DGHs who have little opportunity for practical clinical training, and trainees in university and postgraduate hospitals who see mainly routine patients, without the benefit of individual instruction on complex cases.

To facilitate the development of a purchaser/ provider relationship for PGME, a more structured approach to training will be required. This will involve the establishment of a clearer definition of what PGME should entail. As part of this, it is important that the needs of different grades are taken into account, as well as the mix of experience in different hospital types.

As the next steps, the Region proposes to take the study forward by disseminating its results to a wider audience in order to stimulate debate, and 
convening one or more consensus conferences to examine how a more structured approach to PGME could be implemented. The consensus conferences will be aimed at purchasers and providers of PGME as well as the NHS Executive and

\section{References}

1. The Working Group on Specialist Medical Training. Hospital Doctors: Training for the Future (Report). Department of Health, April 1993. medical Royal Colleges and Faculties. In addtion, the region believes that it is important to engage trainees themselves in the process of shaping PGME.

2. The Review Advisory Committee. Review of the Research and Development Taking Place in the London Postgraduate Special Health Authorities (Report). HMSO, London, 1993. 